\title{
Philosophiques
}

\section{Peirce, Charles Sanders, Ecrits logiques, Tiercelin Claudine et Thibaud Pierre (dir.), Oeuvres III, Paris, Cerf, 2006, 395 pages}

\section{Olivier Perru}

Volume 35, numéro 2, automne 2008

URI : https://id.erudit.org/iderudit/000449ar

DOI : https://doi.org/10.7202/000449ar

Aller au sommaire du numéro

Éditeur(s)

Société de philosophie du Québec

ISSN

0316-2923 (imprimé)

1492-1391 (numérique)

Découvrir la revue

Citer ce compte rendu

Perru, O. (2008). Compte rendu de [Peirce, Charles Sanders, Ecrits logiques,

Tiercelin Claudine et Thibaud Pierre (dir.), Oeuvres III, Paris, Cerf, 2006, 395

pages]. Philosophiques, 35(2), 620-626. https://doi.org/10.7202/000449ar d'utilisation que vous pouvez consulter en ligne.

https://apropos.erudit.org/fr/usagers/politique-dutilisation/ 


\section{$620 \cdot$ Philosophiques / Automne 2008}

de voie du milieu entre ces deux possibilités. Mais, bien évidemment, il est discutable que l'éventail contemporain des théories de la représentation mentale se laisse réduire aussi facilement à cette alternative. L'une des faiblesses du propos de Ramsey est par exemple ne pas considérer les théories non classiques (d'obédience connexionniste et neuroscientifique) de la représentation conçue comme modèle : ces dernières peuvent montrer comment la représentation en tant que modèle, simulation ou émulation peut se poser à un niveau de description et d'explication sous-symbolique, et ce, au moyen d'une théorie élaborée de l'indication et de l'information neuronale ${ }^{1}$. On peut aussi trouver paradoxal le fait que l'auteur fasse appel, de manière d'ailleurs peu précise, à nos intuitions populaires à propos de la nature du fonctionnement des représentations afin de critiquer conceptuellement certains modèles scientifiques de la représentation mentale, alors même qu'il conçoit de manière potentiellement éliminativiste les rapports entre les sciences cognitives et la psychologie populaire.

PIERRE STEINER

Université de Technologie de Compiègne

\section{Peirce, Charles Sanders, Ecrits logiques, Tiercelin Claudine et Thibaud Pierre (dir.), CEuvres III, Paris, Cerf, 2006, 395 pages.}

Ce volume est le troisième de l'édition française des œuvres de Charles Sanders Peirce (1839-1914). Initiée en 2002, cette édition comprendra dix volumes, chaque volume regroupant des traductions d'articles de l'auteur autour des axes principaux de son œuvre. C'est ainsi que ce projet met en valeur les travaux de Peirce autour du pragmatisme (I et II), de la logique et de ses rapports avec les mathématiques et la philosophie (III, IV et V), de la sémiotique (VI), de la philosophie de l'esprit (VII), de l'épistémologie (VIII et IX) et de la métaphysique (X). L'œuvre de Peirce est particulièrement difficile; les écrits logiques dont il est question ici, pour méconnus qu'ils soient en France, n'en constituent pas moins un point de passage obligé dans l'entreprise d'édification de la logique formelle aux $\mathrm{XIX}^{\mathrm{e}}$ et $\mathrm{XX}^{\mathrm{e}}$ siècles. Le propos de Peirce était triple : revisiter la syllogistique aristotélicienne à la lumière d'une formalisation plus mathématique, expliciter et interpréter l'œuvre de Boole et son utilité dans la formalisation du discours, proposer un formalisme logique des prédicats et des relatifs, pouvant donner lieu à l'invention de formes graphiques; l'invention des graphes existentiels est une innovation de Peirce qui exprime des modes de combinaison des idées autour des propriétés d'objets individuels. Indépendante de la logique péanorussellienne, l'œuvre logique de Peirce a pourtant apporté de nombreux éléments pour constituer la logique standard contemporaine, et elle est à bien des égards plus complexe; elle passe en revue les grands problèmes de formalisation, classes, relations, prédicats, elle assume d'ailleurs les prédicats dans des formes relationnelles assez complexes et introduit la trivalence des propositions. Toutefois, cette logique de Peirce suppose un développement mathématique ardu, en particulier autour de l'algèbre booléenne, et son usage, dans la notation et l'interprétation du discours, s'avère difficile.

1.Voir par exemple Rick Grush, "The Emulation Theory of Representation: Motor Control, Imagery, and Perception ", Behavioral and Brain Sciences, 2004, 27, 377-442. 
Dans le développement qui suit, nous signalons et discutons brièvement quelques points essentiels de cette traduction française des articles logiques de Peirce, points qui montrent le caractère innovant et spécifique de cette œuvre : transformation et renouvellement de la syllogistique, relecture et interprétation de l'œuvre de Boole, établissement d'une algèbre de la logique, réflexion sur le signe (relation ternaire signe - objet - esprit), et enfin, essai de logique symbolique par l'invention de formes graphiques de la logique.

Peirce examine les syllogismes en s'attachant d'abord aux réductions à la première figure. La présentation adoptée souligne que les divers syllogismes de chaque figure sont des variations de la même inférence, en qualité (affirmation - négation) et en quantité (singulier, particulier - universel), tout en étant réductible à la première figure. Une originalité de Peirce consiste à y introduire un raisonnement probabiliste. Pour le syllogisme de la première figure, on obtient :

La proportion $\mathrm{r}$ des $\mathrm{M}$ possède le caractère aléatoire $\Pi$;

Ces $\mathrm{S}$ sont tirés au hasard à partir des $\mathrm{M}$;

Probablement et approximativement, la proportion $\mathrm{r}$ des $S$ possède le caractère $\Pi$ (p. 32) ${ }^{1}$.

Cette inférence introduit un caractère probabiliste dans le syllogisme classique en Barbara, Tout $M$ est $\Pi$, Tout $S$ est $M$, donc Tout $S$ est $\Pi$. En fait, Peirce introduit une hypothèse probabiliste sur la majeure, puisqu'il ne s'agit pas de «tout $\mathrm{M}$ » mais d'une certaine proportion de M, laquelle proportion est supposée se retrouver dans le tirage aléatoire des $\mathrm{M}$, ce qui justifie le caractère aléatoire de la conclusion. Ce raisonnement est nouveau, dans la mesure où il introduit le caractère probabiliste au cœur du syllogisme à la fois dans la majeure et dans la mineure, ce qui permet de poser " probablement et approximativement » la conclusion (p. 32). On retrouve d'autres exemples dans les pages suivantes. Le même problème, transposé dans la $3^{\mathrm{e}}$ figure, donne:

Les $\mathrm{S}$ sont tirés au hasard à partir des $\mathrm{M}$;

De ces $S$, la proportion $\mathrm{p}$ possède le caractère aléatoire $\Pi$;

Probablement et approximativement, la proportion $\mathrm{p}$ des $\mathrm{M}$ possède le caractère $\Pi$ (p. 35-36).

De façon intéressante, Peirce reconnaît la formule de l'induction dans cette formulation. Mais il ne précise pas qu'il s'agit là de l'induction quantitative, laquelle pourrait effectivement se ramener à ce raisonnement ${ }^{2}$. Mais l'induction aristotélicienne ne se résout pas à ce simple passage du singulier à l'universel. Elle est qualitative et suppose la remontée à un principe et à une cause, notions évidemment absente de l'œuvre

1. Charles Sanders Peirce, "Types of Reasoning", dans Ketner, Kenneth Laine, dir., Reasoning and the Logic of Things: the Cambridge Conferences Lectures of 1898, Cambridge, Massachusetts, Harvard University Press, 1992.

2. Chez Peirce, l'induction est un raisonnement statistique permettant une universalisation, ce qui correspond bien à l'exemple du syllogisme que nous avons donné. L'abduction, qui semble peut-être mieux correspondre au sens habituel de la démarche inductive à partir de données expérimentales, consiste à remonter à une hypothèse plausible au terme d'une inférence. Pour un bref résumé sur la question, voir : Raymond Robert Tremblay, "Charles Sanders Peirce », [en ligne][http ://www.cvm.qc.ca/encephi/contenu/philoso/peirce.htm] 
de Peirce qui se situe dans le champ de la logique du signe, dans celui des mathématiques ou encore de la sémiotique. Peirce se réclame parfois d'Aristote, mais le lecteur verra tout de suite qu'il dépend en réalité de la scolastique et du nominalisme, lesquels ont mis la logique au premier plan (cf. p. 17). Le propos de Peirce est clair: fonder la métaphysique sur la logique, et la logique sur les mathématiques. On n'est donc pas dans l'optique d'une philosophie interrogeant sur l'homme et sur la nature, mais au seuil d'une entreprise de formalisation de l'existant, du discours, de l'environnement.

Le travail entrepris sur les syllogismes conduit l'auteur à des substitutions. Par exemple, si on définit «quelque $-S$ » comme « la partie de $S$ qui est ou n'est pas $P$ lorsque quelque $S$ est ou n'est pas $\mathrm{P}$ » (p. 65) ${ }^{3}$. On peut alors transformer les syllogismes Disamis et Bocardo,

Quelque S est (ou n'est pas) P;

Tout $\mathrm{S}$ est $\mathrm{M}$;

Quelque M est (ou n'est pas) P (p. 65).

dans les formes suivantes:

Tout quelque $\Pi$ S est ou n'est pas P;

Quelque $M$ est quelque $\Pi S$;

Quelque M est (ou n'est pas) P (p. 66).

C'est une réduction à la première figure, mais en introduisant " quelque $-S$ ", c'est-à-dire l'élément de $S$ justifiant ou contredisant l'affirmation de P. En fait, l'auteur entreprend tout un travail sur les formes des syllogismes en cherchant entre autres à préciser les particulières au sens d'Aristote, qui deviennent chez lui des propositions singulières (tendance déjà présente chez les nominalistes et dans la Logique de PortRoyal). Ainsi, le syllogisme précédent se résumerait ainsi :

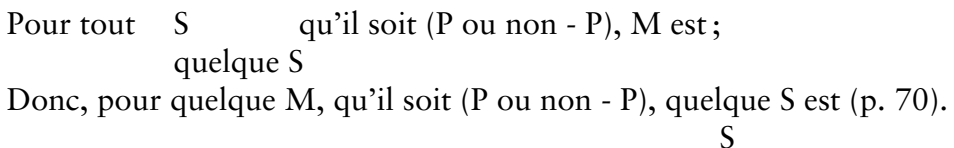

Ce travail présente l'intérêt de conduire Peirce à une redéfinition de l'extension et de la compréhension d'un terme. Peirce veut renouveler ces définitions par rapport aux logiciens de Port-Royal et à De Morgan. Il adopte donc les termes de «largeur » et de "profondeur » pour l'extension et la compréhension. La "largeur informée » d'un terme signifie «toutes les choses réelles dont il est prédicable avec vérité logique dans l'ensemble, dans un état supposé d'information» (p. 93). La "profondeur informée » signifie" tous les caractères réels (par opposition à de purs et simples noms) qui peuvent en être prédiqués (avec vérité logique dans l'ensemble), dans un état supposé d'information" (p. 93). Le caractère intentionnel de cette saisie de l'extension et de la compréhension est affirmé dans la relativité à « un état supposé d'information ». La largeur et la profondeur informée sont distinguées en essentielles et substantielles, ce qui apporte une distinction entre intentionnalité et réalité dans l'approche

3. Charles Sanders Peirce, "On the Natural Classification of Arguments", Proceedings of the American Academy of Arts and Sciences, vol. 7, 9 avril 1867, p. 261-287. 
par sujet et prédicat. La profondeur essentielle englobe les «qualités réellement concevables qui en sont prédiquées dans sa définition" (p. 94). Si T est à la fois P', P” et P'", cela résume toute sa signification mais celle-ci n'implique pas que T existe. On se situe dans l'intention de signification. La «largeur essentielle» comprend les « choses réelles» dont un terme est prédicable, mais cela implique que non - T ait aussi une largeur essentielle. Pour expliciter ces définitions, l'auteur montre que « être » n'a aucune largeur essentielle et que « rien » n'a aucune profondeur essentielle :

On ne peut énumérer les sujets essentiels de l'être pas plus que les prédicats essentiels de rien (p. 95).

Plus pertinentes sont sans doute les définitions de la largeur substantielle et de la profondeur substantielle, qui sont supposées se situer dans le réel. On pourrait croire qu'il y a un reste de réalisme dans ces définitions, mais Peirce définit ces termes dans l'ordre du discours afin de refonder des catégories du langage.

La largeur substantielle est l'agrégat des substances réelles dont seul un terme est prédicable avec une absolue vérité. La profondeur substantielle est la forme concrète réelle qui appartient à tout ce dont un terme est prédicable avec une absolue vérité (p. 95).

Cette affirmation suppose l'appréhension des sujets-substances correspondant à une classe et donc prédicables par un terme ainsi que l'appréhension d'une forme concrète inhérente au sujet dont on parle. L'insistance sur le terme comme critère de vérité et sur l'individualisation des substances-sujets semble renvoyer à des thèmes nominalistes. À partir de ce fondement, Peirce s'efforce d'ébaucher de nouvelles catégories.

Un apport essentiel de Charles Sanders Peirce est sa relecture et son interprétation de l'œuvre de Georges Boole. L'application de signes algébriques à la logique est un préalable, et ces signes de l'addition et de la multiplication logique servent à fonder une langue artificielle. Ainsi, en posant $\mathrm{m}=$ man (homme) et $\mathrm{b}=$ black (noir),

$\mathrm{m}+\mathrm{b}$ est mis pour tous les hommes et toutes les choses noires, sans impliquer en aucune façon que les choses noires doivent être prises en plus des hommes (p. 129) $)^{4}$.

C'est la réunion des deux ensembles. La multiplication logique permet de développer une logique des relations: "lw» dénote tout ce qui est amoureux (l) d'une femme $(w),(1+s) w$, tout ce qui est amoureux ou serviteur d'une femme, $(\mathrm{sl}) \mathrm{w}$,

4. Charles Sanders Peirce, « Description of a Notation for the Logic of Relatives, Resulting from an amplification of the Conceptions of Boole's Calculus of Logic ", Communication, 26 janvier 1870, Writings of Charles S. Peirce: A Chronological Edition, Indiana University Press, 1977, 2, p. 359-429. 


\section{Philosophiques / Automne 2008}

tout ce qui est serviteur d'un amoureux d'une femme ${ }^{5}$. Plus subtil : si la relation g désigne "donateur à - de - ", on écrira $\mathrm{g}^{\mathrm{xy}}$ pour "donateur à x de y» (p. 131). Si le produit oh désigne tous ce qui est propriétaire $(\mathrm{o})$ d'un cheval $(\mathrm{h}), \mathrm{g}^{\mathrm{oh}}$ signifiera donateur d'un cheval (h) à un propriétaire (o). On n'évite pas alors une difficulté : dans le cas de tel cheval individuel, il peut s'agir du donateur d'un cheval au propriétaire de ce cheval, ce qui plaide pour une notation indicielle clarifiant cette relation. Pour exprimer « donateur d'un cheval à un amoureux d'une femme ", et donc assumer deux types de relation à la fois, Peirce renonce à écrire directement $\mathrm{g}(\mathrm{lw}) \mathrm{h}$ pour ne pas abandonner le principe associatif de la multiplication logique et il appose des indices aux lettres pour exprimer les relations. C'est l'invention de «marques de références » d'un usage sans doute trop complexe pour se repérer dans les termes de la relation (p. 133). La fonction puissance sert à introduire l'universalité dans la relation : $\mathrm{b}^{\mathrm{a}}$ dénote "traître avec tout ennemi » et $\mathrm{l}^{\mathrm{w}}$, " amoureux de toute femme » (p.131). Nous ne pouvons pas développer toutes les notations de Peirce en logique des prédicats et des relations, il faut se contenter de noter qu'elles devancèrent les travaux de Frege en la matière et qu'elles reposent sur un substrat mathématique supposant l'addition et la multiplication logique au sens de Boole 6

Ainsi, Charles Sanders Peirce en vint à établir une " algèbre de la logique » (1880), reformalisant les syllogismes en assimilant « est » à la copule, et selon le sens de la proposition logique, à l'implication ou à l'identité (p. 195-249) ${ }^{7}$. Cette algèbre de la logique est basée sur les travaux antérieurs de De Morgan (1846). Dans son article de 1870 sur la notation, Peirce introduisait le signe "plus petit que ", au sens où " $\mathrm{f}<\mathrm{m}$ signifie que tout Français est un homme mais qu'il y a des hommes en dehors des Français » (p. 127). Si l'on écrit $\mathrm{f}-<\mathrm{m}$, ce signe étant pris au sens algébrique de $\leq$, on veut

5. En fait, d'après l'article d'Eric Hammer qui a fait une synthèse de la notion de relation chez Peirce, dans Stanford Encyclopedia of Philosophy, l'utilisation que Peirce fait de la multiplication logique dans les pages que nous commentons concernerait le produit relatif. Voici comment Hammer donne sur des exemples les définitions de Peirce de la somme, du produit, du produit relatif:

"Given relative terms such as "friend of" and "enemy of" (more briefly "f" and " $e$ "), Peirce studied various operations on these terms such as the following:

(union) friend of or enemy of. A pair $<\mathrm{a}, \mathrm{b}>$ stands in this relation if and only if it stands in one or both of the relations. In symbols " $\mathrm{f}+\mathrm{e}$ ".

(intersection) friend of and enemy of. A pair $<\mathrm{a}, \mathrm{b}>$ stands in this relation if and only if it stands in both of the relations. In symbols "f. e".

(relative product) friend of an enemy of. A pair $<\mathrm{a}, \mathrm{b}>$ stands in this relation if and only if there is a c such a is a friend of $c$ and $c$ is an enemy of b. In symbols " $\mathrm{f}$; $\mathrm{e}$ " ". Eric Hammer, «Peirce's logic », Stanford Encyclopedia of Philosophy, Stanford University, 2007.

6. À propos de l'article que nous venons d'évoquer, Robert Burch écrit: «In 1870, Peirce published a long paper Description of a Notation for the Logic of Relatives in which he introduced for the first time in history, two years before Frege's Begriffschrift a complete syntax for the logic of relations of arbitrary adicity (or: arity). In this paper, the notion of variable was invented and Peirce provided devices for negating, for combining relations (basically by building upon De Morgan's relative product and relative sum), and for quantifying existentially and universally ». Robert Burch, "Charles Sanders Peirce ", Stanford Encyclopedia of Philosophy, Stanford University, 2006.

7. Charles Sanders Peirce, "On the Algebra of Logic", The American Journal of Mathematics 3, 1880, p. 15-57, Writings of Charles S. Peirce: A Chronological Edition, Indiana University Press, 1982, 4, p. 163-209. 
signifier que tout Français est un homme "sans dire s'il y a ou non d'autres hommes » (p. 127). Dans le texte de 1880, Sur l'algèbre de la logique, Peirce reconsidère A $-<\mathrm{B}$ au sens de $\mathrm{A}<\mathrm{B}$; A étant donc inclus dans B, tout $\mathrm{A}$ est $\mathrm{B}$ et $\mathrm{A}$ implique B. Par contre, si $\mathrm{B}>\mathrm{A}$, alors $\mathrm{B}$ n'est pas forcément $\mathrm{A}$ (il peut y avoir des $\mathrm{B}$ qui ne sont pas $\mathrm{A}$ ), $B-<A$. Dans le cas de l'identité, il y a double implication: $\mathrm{A}-<\mathrm{B}$ et $\mathrm{B}-<\mathrm{A}$, donc $\mathrm{A}=\mathrm{B}$.

Peirce va retraduire toute la syllogistique héritée d'Aristote dans ces conventions et surtout, il étendra la signification du syllogisme en introduisant un terme singulier dans la mineure; il demeure fidèle en cela à la pratique issue d'Occam et de la Logique de Port-Royal. Ainsi, à partir du syllogisme en Barbara, j'écris: Tous les hommes sont mortels, tous les patriarches sont des hommes, alors tous les patriarches sont mortels. On peut introduire une proposition singulière dans la conclusion de ce syllogisme, ce que fait Peirce:

Si tous les patriarches sont mortels, [alors] la Bible se trompe (p. 209).

En repartant de la mineure, il écrit :

Tous les patriarches sont des hommes,

Si tous les patriarches sont mortels, la Bible se trompe;

Si tous les hommes sont mortels, la Bible se trompe (p. 209).

En fait, l'astuce consiste à introduire une proposition singulière « la Bible se trompe ", implicitement contenue dans l'universelle "tous les patriarches sont mortels ". L'introduction d'un cas singulier est comme un quatrième terme du syllogisme, appelé par Peirce, le syllogisme indirect majeur. En réalité, l'algèbre de la logique introduit l'hypothèse et l'implication dans le syllogisme, c'est donc une transformation des raisonnements syllogistiques en formes implicatives hypothétiques. Il serait trop fastidieux de passer en revue cette logique qui lie l'addition logique, la multiplication logique, mais on peut affirmer le caractère novateur de la présentation de l'implication et de son rôle dans le raisonnement.

Au-delà des règles de l'algèbre de la logique et plus fondamentalement, Peirce a réfléchi sur la nature du signe en tant que notation.

Un signe est en relation conjointe avec la chose dénotée et avec l'esprit (p. 279) ${ }^{8}$.

Il propose la relation ternaire signe - objet - esprit, le signe renvoyant à la fois à l'objet et à l'esprit. Il existe, selon Peirce, trois types de signes, les icônes (comme les cercles dans les diagrammes d'Euler), les tokens (comme les ombres rajoutées par Venn dans les diagrammes), les indices. Les indices relient la vérité énoncée dans les autres signes à des objets et permettent aussi de " "distinguer" le monde réel d'un monde de l'imagination" (p. 281). Puis, Peirce examine les icones fondamentales de l'algèbre: le principe d'identité, la règle "selon laquelle les divers antécédents d'une consequentia peuvent être transposés » (p. 288), enfin le "principe de la transitivité de la copule» (p. 289). En outre, Peirce développe une logique des relatifs de première intention.

Signalons enfin les travaux innovants de cet auteur, encore assez méconnu en France, sur les formes graphiques de la logique. Certains commentateurs y voient la

8. Charles Sanders Peirce, "On the Algebra of Logic. A contribution to the philosophy of notation ", The American Journal of Mathematics 7, 2, 1885, p. 180-202, Writings of Charles S. Peirce: A Chronological Edition, Indiana University Press, 1982, 5, p. 162-190. 
partie la plus inventive, voire la plus géniale, de l'œuvre de Peirce. Il s'agit d'un essai de logique symbolique (formes graphiques de la logique), placé en quatrième partie de cet ouvrage regroupant huit petits textes de Peirce, écrits à la fin de sa vie, plus précisément entre 1902 et 1906 . Nous ne commentons pas cette partie, bien qu'elle soit peut-être l'aboutissement de toute la pensée logique de Peirce : elle demanderait en ellemême une étude critique distincte.

Cette présentation française de l'œuvre de Peirce est d'une grande richesse : dans ce troisième volume des CEuvres, Claudine Tiercelin et Pierre Thibaud mettent à la disposition des logiciens et des épistémologues en général une traduction et des annotations d'une grande qualité. Ce travail est essentiellement destiné à des spécialistes, encore que sémioticiens, philosophes, mathématiciens puissent être intéressés par tel ou tel article de ce volume, tant est vaste le domaine interdisciplinaire qui fit l'objet des recherches de Peirce. La lecture de ce volume s'avère néanmoins ardue et suppose une solide culture logique et mathématique et une interrogation sur le fondement et le sens des formalisations en logique. 